\section{Prevalência do beber e dirigir em Belo Horizonte, Minas Gerais, Brasil}

\author{
Drinking-and-driving prevalence in Belo Horizonte, \\ Minas Gerais State, Brazil
}

\begin{abstract}
Problems resulting from alcohol consumption by drivers have been studied worldwide, and epidemiological research points to high injury and death rates related to drinking-and-driving. However, equivalent data are limited in Brazil. In this study, 913 drivers were stopped on public roads with heavy traffic and high concentrations of bars, restaurants, and nightclubs in Belo Horizonte, Minas Gerais State, and asked to answer a questionnaire and submit to an active breathalyzer test. The study was done in December 2005 and December 2006. The study adopted the internationally accepted sobriety checkpoint method. In the sample, 38.0\% of drivers showed some trace of alcohol in their exhaled air, and 19.6\% were at or above the legal limit $(0.6 \mathrm{~g} / \mathrm{l})$. These figures were five times those found in similar surveys in other countries. The findings suggest a critical drinking-and-driving problem in Belo Horizonte (and probably elsewhere in Brazil) and the need for on-going research, the development of specific public policies to deal with the problem, and effective enforcement of the existing law.
\end{abstract}

Alcohol Drinking; Automobile Driving; Legislation
Valdir Ribeiro Campos ${ }^{1}$

Rogério Salgado 1

Mariela Campos Rocha 1

Sérgio Duailibi 2,3

Ronaldo Laranjeira 2,3

\section{Introdução}

A Organização Mundial da Saúde (OMS) estima que existam 2 bilhões de consumidores de bebidas alcoólicas em todo o mundo e, desses, 76,3 milhões com diagnóstico de transtornos relacionadas ao uso de álcool. Os índices de morbidade e mortalidade relacionados a essa realidade são consideráveis ${ }^{1}$. Os acidentes automobilísticos ocupam papel proeminente nas estatísticas, pois, em todo o mundo, entre um quarto e a metade dos acidentes de trânsito com vítimas fatais estão associados ao uso do álcool por algum dos responsáveis pela ocorrência 2 .

A bebida proporciona aos motoristas um falso senso de confiança, prejudicando habilidades como: a atenção, a coordenação, a acuidade visual e o julgamento de velocidade, tempo e distância 3. Decréscimos no desempenho aparecem, até mesmo, na ingestão de um único drinque, apesar de freqüentemente a pessoa ficar certa de que seu desempenho, na verdade, melhorou 4. Mesmo quantidades pequenas de álcool, abaixo dos limites legais, aumentam as chances de ocorrerem acidentes ${ }^{5}$. Por exemplo, em adolescentes, o risco de acidentes aumenta após apenas uma dose de bebida, dobra após duas e aumenta em dez vezes após cinco doses 5 . Essa condição é extremamente preocupante em motoristas jovens - que têm 55 vezes mais chances de se envolver em acidentes de trânsito do que um indivíduo de 65 anos - devido a alguns fatores como: a pouca 
experiência como condutor, a impulsividade, o hábito de dirigir em alta velocidade e o uso menos freqüente do cinto de segurança ${ }^{2}$. A maioria dos acidentes fatais, relacionados ao uso de bebida alcoólica e direção, ocorre na faixa etária dos 21 aos 24 anos, e $80 \%$ deles ocorrem no período das 20 às 4 horas da manhã das noites dos fins de semana 6,7 . O álcool diminui a eficiência cerebral e reduz a visão noturna em $25 \%$ e o tempo de reação em até $30 \%$. Esses efeitos são mais intensos quanto menor a tolerância ao álcool 8 . Diversos estudos têm mostrado a associação entre os acidentes de trânsito envolvendo jovens e o ato de beber e dirigir e têm também sugerido medidas de regulação desse comportamento para redução dos riscos de acidentes de trânsito 9,10,11. Desde a década de 80, iniciou-se em vários países, notadamente nos Estados Unidos, uma série de mudanças na legislação, resultando em ações preventivas e coibitivas relacionadas ao ato de dirigir alcoolizado. Estudo realizado por Lund \& Wolfe 12 , sobre a mudança na incidência do beber e dirigir nos Estados Unidos, entre 1973 e 1986, apontou uma queda de um terço ou mais nas taxas de acidentes fatais após a adoção do bafômetro.

A importância do "beber e dirigir" pode ser demonstrada pelos elevados custos sociais do consumo de bebidas alcoólicas e suas conseqüências para os acidentados, podendo ser considerado um problema de saúde pública nos países em desenvolvimento, considerando-se o pesado fardo econômico-social resultante da soma dos prejuízos materiais, da assistência à saúde $\mathrm{e}$ referentes à perda de produtividade ${ }^{8}$. No Brasil, os poucos estudos existentes apontam o beber e dirigir como um problema relevante. Apesar de quase não possuir dados epidemiológicos da ocorrência de acidentes de trânsito relacionados ao uso de bebidas alcoólicas, o Brasil é um dos países com maiores proporções de problemas relacionados a esse consumo, com imensos custos sociais. Pesquisa encomendada pelo Governo Federal, sobre os custos dos acidentes de trânsito, mostra, em seus resultados preliminares, que $53 \%$ dos acidentados no trânsito, atendidos no Ambulatório de Emergência do Hospital das Clínicas, em São Paulo, num determinado período, estavam com índices de alcoolemia superiores aos permitidos pelo Código de Trânsito Brasileiro, sendo em sua maioria pessoas do sexo masculino com idade entre 15 e 29 anos 13 .

Pesquisa realizada em Belo Horizonte, com pacientes - vítimas de acidentes de trânsito - atendidos em três hospitais da capital, apontou que $15 \%$ de todos os envolvidos haviam consumido bebidas alcoólicas nas 8 horas anteriores ao acidente, sendo que os motoristas tiveram o maior percentual $(27,7 \%)$ de respostas positivas para o consumo de bebidas alcoólicas. Foi constatado, ainda, que o período de maior ocorrência de acidentes automobilísticos é o de 0 às 6 horas da manhã, principalmente, nos sábados e domingos, apesar do volume de tráfego ser bem menor nesses dias 14 .

Assim, este artigo visa identificar, na amostra estudada, padrões de comportamento em relação ao beber e dirigir em Belo Horizonte. Os resultados desta pesquisa são os primeiros dados coletados através da metodologia do uso do bafômetro de forma aleatória, em uma amostra, em uma capital do país.

\section{Metodologia}

O levantamento de dados foi realizado no mês de dezembro de 2005 e dezembro de 2006, nas noites de sexta-feira e sábado, no horário de 22 às 3 horas da madrugada, em "postos de fiscalização de sobriedade" (sobriety checkpoint), estabelecidos em vias públicas consideradas estratégicas e de tráfego mais intenso, com maiores concentrações de bares, restaurantes e casas noturnas.

Os pontos de checagem foram previamente estabelecidos, de acordo com mapeamento da Empresa de Transportes e Trânsito de Belo Horizonte (BHTRANS), e envolveu a participação da polícia de trânsito, uma equipe de 30 pesquisadores, 2 supervisores, 2 coordenadores e pessoal de apoio da Sub-Secretaria Estadual Antidrogas de Minas Gerais. Desse modo, uma vez escolhidos, aleatoriamente, os veículos pelo coordenador do grupo, os motoristas eram parados por policiais do comando de trânsito, devidamente equipados e identificados, que desviavam os veículos para o ponto de checagem e verificavam as condições de segurança do tráfego, dos condutores, dos passageiros e de toda a equipe de pesquisadores, supervisores, coordenadores e pessoal de apoio envolvidos no trabalho de abordagem, evitando, assim, riscos de assalto, atropelamento e acidentes. Em seguida, o pesquisador explicava a cada motorista o motivo da parada, informando tratar-se de uma pesquisa educativa, e os que concordavam em participar assinavam um Termo de Consentimento Livre e Esclarecido e recebiam um folheto educativo sobre a relação álcool e direção.

Após esse procedimento, aquele condutor que aceitasse participar da pesquisa respondia um questionário anônimo sobre: os dados sócioeconômicos e demográficos, comportamento quanto ao padrão do uso de bebidas alcoólicas, comportamento quanto ao padrão do uso de bebidas alcoólicas e direção, conhecimento sobre 
parte da lei que rege esses assuntos no trânsito e opinião sobre o uso do bafômetro. Em seguida, o condutor era convidado a se submeter ao teste do bafômetro ativo, o que lhe era assegurado de que os dados auferidos no bafômetro não seriam compartilhados com a polícia. Após a aceitação em submeter-se ao teste, o coordenador da equipe explicava ao condutor o funcionamento do aparelho, e os valores nele obtidos eram devidamente registrados no questionário aplicado.

Por fim, o entrevistador realizava uma avaliação do estado geral do condutor (classificandoo como: normal, sob efeito de álcool ou outras drogas, mas sem embriaguez, ou visivelmente embriagado), do tipo de veículo, do número de passageiros e do uso de equipamentos de segurança. Esses procedimentos tiveram, em média, a duração de cinco minutos, e algumas medidas de segurança foram adotadas em relação aos motoristas com níveis de álcool igual ou superior ao limite estabelecido por lei. Foram disponibilizados 10 motoristas profissionais da Sub Secretaria Antidrogas de Minas Gerais, que estiveram o tempo todo à disposição, para conduzir às suas residências os entrevistados que apresentassem teor alcoólico incompatível com a direção. E aquele que recusasse a assistência de um motorista ou era orientado a trocar de direção com o acompanhante, caso este reunisse as condições legais para conduzir o veículo, ou lhe era sugerido aguardar juntamente com a equipe até estar em melhores condições de conduzir o veículo, ou ainda era aconselhado a solicitar alguém em perfeitas condições para que pudesse vir buscar o veículo.

Além do mais, os materiais e os métodos referentes ao uso dos bafômetros - manuseio adequado, condicionamento, baterias e fontes de eletricidade, limpeza e esterilização de bicos utilizados - foram, também, objeto de adequada instrução durante a etapa de treinamento das equipes, incluindo a aplicação do questionário.

Foi verificado através deste estudo a aplicabilidade e aceitabilidade do bafômetro na coleta de dados. E esta pesquisa, ainda, pretende auxiliar na configuração de desenhos para pesquisas e orientar intervenções na área do ato de beber e dirigir.

\section{Resultados}

\section{Análise estatística}

Foi utilizado como banco de dados e instrumento de análise estatística o aplicativo SPSS (SPSS Inc., Chicago, Estados Unidos). A pesquisa foi conduzida dentro dos padrões da Declaração de Helsinki e aprovada pela comissão de ética da Escola Paulista de Medicina, Universidade Federal de São Paulo.

\section{Participação na pesquisa}

Foram entrevistados 990 condutores, distribuídos entre carros, motocicletas e utilitários, sendo que 913 condutores $(92,2 \%)$ aceitaram participar em pelo menos uma das etapas da pesquisa (questionário e bafômetro ativo).

\section{Dados sócio-econômicos e demográficos}

A distribuição por sexo e faixa etária estabeleceu uma relação de 4 homens para cada mulher e uma predominância da faixa de 18 a 30 anos (55,5\%) respectivamente. Quanto ao estado civil, houve uma maior freqüência de solteiros $(66,6 \%)$. O grau de instrução concentrou-se na faixa dos que possuem nível superior completo ou incompleto $(76,8 \%)$, dos que têm emprego formal e renda familiar acima de 8 salários mínimos (73,7\%), coincidindo com o perfil sócio-econômico dos moradores da região pesquisada.

\section{Dados referentes ao padrão de consumo de bebidas alcoólicas}

O consumo de bebidas alcoólicas durante a semana foi assim distribuído: $60,4 \%$ declararam beber 1 a 2 dias por semana, mantendo este mesmo percentual na faixa etária dos 18 aos 30 anos, $8,4 \%$ bebem 3 a 4 dias por semana, 1,9\% bebem todos os dias da semana, 2,8\% bebem de 15/15 dias ou não têm periodicidade e $2,1 \%$ raramente fazem uso de bebidas alcoólicas. As bebidas mais usadas são: a cerveja (51,8\%), o vinho $(11,2 \%)$, os destilados - uísque, vodca, conhaque e pinga $(10,8 \%)$, e as bebidas tipo ice $(1,3 \%)$, com a quantidade de uso em média: de 2 a 3 copos ou latas de cerveja/chope, 1 a 3 copos/taças de vinho e de uma a duas doses de destilados. Dos que participaram da pesquisa, 368 (40,3\%) declararam ter ingerido bebida alcoólica no dia da entrevista e $330(36,1 \%)$ recusaram o teste do bafômetro. 
Dados referentes ao comportamento no trânsito

As informações sobre o envolvimento em acidentes de trânsito, as medidas adotadas quando os motoristas bebem, a avaliação do estado geral do condutor, realizada pelo entrevistador, e o conhecimento da legislação de trânsito, em relação ao uso de álcool, por parte dos motoristas, apresentaram os seguintes dados: 334 (36,6\% dos entrevistados) declararam já ter se envolvido em acidentes de trânsito como motoristas, sendo que, entre estes, 200 (60\%) tem o padrão de consumo de álcool de dois dias por semana e 185 $(55,4 \%)$ tem a predominância na faixa etária dos 18 aos 30 anos. Dentre as atitudes que os entrevistados adotam quando bebem, a maioria 544 $(59,6 \%)$ informou que entrega o veículo a outro motorista, ou pega um ônibus, um táxi ou uma carona, e apenas 209 (22,9\%) admitiram considerar que a bebida não atrapalha ao dirigir e adotam medidas de proteção, como: tomar café, dirigir bem devagar e ter mais atenção quando faz uso de álcool. Na avaliação sobre o estado geral do condutor, $14 \%$ dos motoristas estavam visivelmente sob efeito de álcool ou de outras drogas com ou sem embriaguez. Na verificação dos conhecimentos sobre a legislação de trânsito em relação ao uso de álcool, os entrevistados consideraram mais grave dirigir alcoolizado $(51,8 \%)$ e apenas $13,1 \%$ souberam informar corretamente o teor de álcool no sangue estabelecido pelo Código de Trânsito Brasileiro para que uma pessoa seja impedida de dirigir $(0,6 \mathrm{~g} / \mathrm{l}) 15$.

\section{Aceitação do bafômetro ativo}

Dos 913 entrevistados que aceitaram participar da pesquisa respondendo ao questionário, 83,8\% declararam ser favoráveis ao uso do bafômetro, e $579(63,4 \%)$ aceitaram ser submetidos ao teste. Os resultados obtidos apontam que $19,6 \%$ dos entrevistados dirigiam com níveis de álcool iguais ou superiores aos limites legais $(0,6 \mathrm{~g} / \mathrm{l})$, e $18,4 \%$ apresentavam algum índice de álcool no ar expirado, portanto, $38 \%$ dos condutores dirigiam com algum nível de álcool no sangue. Dentre esses, apenas 45 entrevistados (20,5\%) foram identificados pelos entrevistadores como estando sob efeito de bebidas alcoólicas ou outras drogas (Figura 1).

\section{Discussão}

Os problemas decorrentes do consumo de álcool em motoristas são estudados internacionalmente, e estudos epidemiológicos indicam alta prevalência de morbidade e mortalidade relacionadas ao beber e dirigir. A OMS sugere quatro ações para controlar os problemas relacionados ao uso de bebidas alcoólicas e direção: redução dos limites de alcoolemia para dirigir, estabelecimento de postos de fiscalização de sobriedade com uso de bafômetro, suspensão administrativa da licença de motoristas intoxicados e graduação do licenciamento para motoristas novatos.

No Brasil, existem escassos dados nacionais a respeito dos padrões de comportamento em relação ao ato de beber e dirigir. Este estudo é um dos pioneiros a apresentar dados sobre tais padrões de comportamento numa grande capital do país, obtidos através da metodologia do estabelecimento de postos de checagem de sobriedade com o uso de bafômetros. Os resultados apontam que mais da metade dos entrevistados é jovem, de 18 a 30 anos, e que mantém um padrão de consumo de bebidas alcoólicas de um a dois dias por semana, com proporção de gênero de quatro homens para cada mulher. A influência das propagandas de bebidas alcoólicas, sob hábitos culturais, se faz perceber através da preferência, por mais da metade dos usuários da substância, pelo consumo do chope e da cerveja - associados com jovens, personalidades do esporte, vida artística, virilidade, sensualidade, diversões nos finais de semana e para encontros em bares, restaurantes e casas noturnas.

Considerando-se que mais de um terço dos entrevistados, que aceitaram responder ao questionário, declararam já ter se envolvido em acidentes de trânsito como motoristas, sendo que, entre esses, a maioria é jovem, com um padrão de consumo de bebidas alcoólicas de um a dois dias por semana, e que o horário e os dias de realização da pesquisa foram favoráveis, assim como o risco de acidentes entre jovens aumenta após qualquer concentração de bebida alcoólica, pode-se dizer que os dados são preocupantes, pois o risco relativo de acidentes automobilísticos, para jovens que dirigem depois de beber, aumenta após apenas uma dose de bebida, dobra após duas e aumenta em dez vezes após cinco doses. A crença de que os condutores que fizeram uso de álcool e se envolveram em acidentes automobilísticos seriam aqueles que fazem uso crônico de álcool ou são dependentes dele não se aplica nesses achados, mas lembra o usuário de álcool bem mais próximo do nosso dia-a-dia. Isso porque, a bebida alcoólica, proporciona aos motoristas um falso senso de confiança preju- 


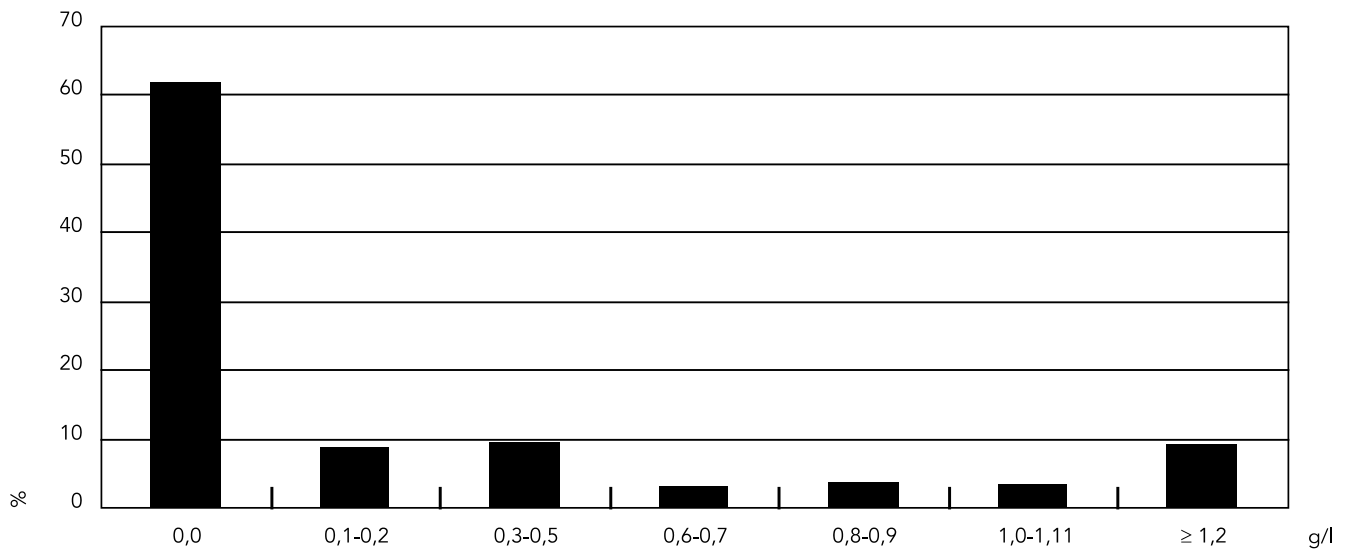

dicando habilidades como a atenção, a coordenação e o tempo de reação, principalmente, no horário noturno.

Apesar de a maioria dos condutores estar consciente sobre os riscos do uso de álcool e direção, ser favoráveis ao uso do bafômetro como instrumento de controle de acidentes de trânsito, de $90 \%$ dos que ingeriram bebidas alcoólicas no dia da entrevista ter recusado o teste do bafômetro e , na percepção dos entrevistadores, estar sob efeito de álcool e outras drogas, o teste do bafômetro, de fato, mostrou-se efetivo em apontar que mais de um terço dos submetidos ao teste (38\%) dirigiam com algum nível de álcool no sangue. A discrepância desses dados pode indicar que mesmo motoristas conscientes sobre o risco de beber e dirigir, aparentemente sóbrios, podem estar intoxicados pelo uso de álcool, com senso subjetivo do beber seguro e certos da ausência de medidas preventivas e coercitivas ao dirigir alcoolizado, oferecendo, assim, risco para si mesmo e para outras pessoas.

Assim, a presente pesquisa, apresentou dados básicos de uma dada região da cidade, contribuindo para o conhecimento, as orientações, as intervenções e as configurações de desenhos de pesquisas futuras a respeito do uso de bebidas alcoólicas e direção. Outros estudos em regiões diversificadas da cidade e interior do estado poderão avaliar melhor a amplitude do problema.

\section{Conclusão}

Os valores encontrados na aplicação do bafômetro ativo acusaram que $19,6 \%$ dos motoristas apresentaram níveis de alcoolemia iguais ou acima dos limites legais, que é de $0,6 \mathrm{~g} / \mathrm{l}$, e 18,4\% apresentaram algum nível de álcool no ar expirado, com um total de $38 \%$ dos que dirigiam com algum nível de álcool no sangue. Esses achados apontam para um índice cinco vezes maior aos encontrados em pesquisas semelhantes realizadas em outros países. Dessa forma, os achados deste estudo sugerem a relevância do problema de beber e dirigir na cidade de Belo Horizonte (e provavelmente no Brasil). Contudo, o objetivo principal deste estudo foi o de fazer o primeiro levantamento de dados, numa grande capital do país, sobre o uso de bebida alcoólica e direção de veículos, com o uso de bafômetros, e o de possibilitar que essas informações venham realçar a necessidade de pesquisas permanentes, do desenvolvimento de políticas públicas específicas para o assunto e do eficaz cumprimento da lei existente. 


\section{Resumo}

Os problemas decorrentes do consumo de álcool em motoristas são estudados internacionalmente, e estudos epidemiológicos indicam alta prevalência de morbidade e mortalidade relacionadas ao beber e dirigir, entretanto existem escassos dados nacionais a respeito. Neste presente estudo, 913 condutores de veículos foram parados, em vias públicas de tráfego com maiores concentrações de bares, restaurantes e casas noturnas, em Belo Horizonte, Minas Gerais, Brasil e solicitados que respondessem a um questionário e fizessem o teste do bafômetro ativo. Esta pesquisa foi realizada no mês de dezembro de 2005 e dezembro de 2006. Para tanto, adotamos a metodologia do tipo pontos de fiscalização de sobriedade utilizada internacionalmente. $\mathrm{Na}$ nossa amostra, 38,0\% dos motoristas apresentavam algum traço de álcool no ar expirado e 19,6\% estavam com níveis de álcool iguais ou acima dos limites legais $(0,6 \mathrm{~g} / \mathrm{l})$. Esses dados foram cinco vezes maiores do que aqueles encontrados em pesquisas semelhantes, em outros países. Os achados deste estudo sugerem a relevância do problema na cidade de Belo Horizonte (e provavelmente no Brasil), a necessidade de pesquisas permanentes, do desenvolvimento de políticas públicas específicas para o assunto e do eficaz cumprimento da lei existente.

Consumo de Bebidas Alcoólicas; Condução de Veículo; Legislação

\section{Referências}

1. World Health Organization. Global status report on alcohol 2004. Geneva: Department of Mental Health and Substance Abuse; 2004.

2. Spinsk I, Laranjeira RR. O fenômeno do dirigir alcoolizado no Brasil e no mundo: revisão da literatura. Rev ABPAPAL 1998; 20:160-5.

3. Royal Society for the Prevention of Accidents. Drinking and driving policy paper. http://www. rospa.com/roadsafety/info/drink_drive.pdf (acessado em 22/Mar/2007).

4. Edwards G. A política do álcool e o bem comum. Porto Alegre: Editora Artes Médicas; 1998.

5. Chou SP, Grant BF, Dawson DA, Stinson FS, Saha T, Pickering RP. Twelve-month prevalence and changes in driving after drinking: United States, 1991-1992 and 2001-2002. Drug Alcohol Depend 2005; 80:223-30.

6. Center for Disease Control and Prevetion. Involvement by young drivers and fatal alcohol relatedmotor-vehicle crash - United States, 1982- 2001. MMWR Morb Mortal Wkly Rep 2002; 51:1089-91.

7. Fell JC, Nash CE. The nature of the alcohol problem in U.S. fatal crashes. Health Educ Q 1989; 16:335-43.

8. Shults RA, Elder RW, Sleet DA, Nichols JL, Alao MO, Carande-Kulis VG, et al. Reviews of evidence regarding interventions to reduce alcohol-impaired driving. Am J Prev Med 2001; 21 Suppl:66-88.

\section{Colaboradores}

V. R. Campos participou da revisão da literatura, planejamento e coordenação da coleta e análise dos dados, discussão dos dados, organização e redação final deste artigo. R. Salgado participou do planejamento e coordenação da coleta e análise dos dados. M. C. Rocha participou da coleta e análise dos dados. S. Duailibi participou da revisão da literatura, discussão dos dados e redação final deste artigo. R. Laranjeira participou do planejamento da pesquisa, análise e revisão do texto final.

\section{Agradecimentos}

Ao apoio da Secretaria de Desenvolvimento Social e de Esportes/Subsecretaria Antidrogas do Governo do Estado de Minas Gerais, através de seu programa de políticas públicas (Intervenção Comunitária para a Redução de Problemas Relacionados ao Consumo de Álcool e Outras Drogas). À Polícia Militar de Minas Gerais, aos acadêmicos de psicologia da Faculdade Metropolitana, aos acadêmicos de psicologia e enfermagem da Fundação Mineira de Educação e Cultura (Universidade FUMEC) e aos residentes de psiquiatria do Instituto de Previdência dos Servidores do Estado de Minas Gerais.
9. Horwood LJ, Fergusson DM. Drink driving and traffic accidents and young people. Accid Anal Prev 2000; 32:805-14.

10. Fergusson D, Swain-Campbell N, Horwood J. Risky driving behaviour in young people: prevalence, personal characteristics and traffic accidents. Aust N Z J Public Health 2003; 27:337-42.

11. Johnson FW, Gruenewald PJ, Treno AJ. Age-related differences in risks of drinking and driving in gender and ethnic groups. Alcohol Clin Exp Res 1998; 22:2013-22.

12. Lund AK, Wolfe AC. Changes in the incidence of alcohol-impaired driving in the United States, 19731986. J Stud Alcohol 1991; 52:293-301.

13. Ministério da Saúde. A política do ministério da saúde para atenção integral a usuários de álcool e outras drogas. Brasília: Ministério da Saúde; 2004.

14. Empresa de Transportes e Trânsito de Belo Horizonte, Secretaria Municipal de Saúde. Pesquisa de levantamento de vítimas de acidentes de trânsito em Belo Horizonte. Belo Horizonte: Prefeitura de Belo Horizonte; 2001.

15. Ministério das Cidades. Código de Trânsito Brasileiro: Lei $n^{\circ} .9503$ de 23 de setembro de 1997. http://www.denatran.gov.br/ctb.htm (acessado em 08/Mai/2007)

Recebido em 15/Mai/2007

Versão final reapresentada em 31/Ago/2007 Aprovado em 24/Set/2007 